\title{
Effectiveness of a preoperative exercise and nutritional support program for elderly sarcopenic patients with gastric cancer
}

\author{
Kazuyoshi Yamamoto ${ }^{1,2}$ (1) Yukiko Nagatsuma ${ }^{3}$ Yasunari Fukuda ${ }^{1,4}$. \\ Motohiro Hirao $^{1}$ - Kazuhiro Nishikawa ${ }^{1}$ - Atsushi Miyamoto ${ }^{1} \cdot$ Masataka Ikeda $^{1}$. \\ Shoji Nakamori ${ }^{1} \cdot$ Mitsugu Sekimoto $^{5} \cdot$ Kazumasa Fujitani $^{5} \cdot$ Toshimasa Tsujinaka $^{6}$
}

Received: 25 October 2016/Accepted: 8 December 2016/Published online: 28 December 2016

(c) The International Gastric Cancer Association and The Japanese Gastric Cancer Association 2016

\begin{abstract}
We established a preoperative exercise and nutritional support program for elderly sarcopenic patients with gastric cancer. Twenty-two gastric cancer patients aged 65 years or older with a diagnosis of sarcopenia according to the algorithm proposed by the European Working Group on Sarcopenia in Older People received our preoperative program. The median duration of the program participation was 16 days. Total calorie and protein intakes were significantly higher after the program than before $[29.4 \pm 6.9 \mathrm{kcal} / \mathrm{kg}$ ideal body weight (IBW) vs $27.3 \pm 5.6 \mathrm{kcal} / \mathrm{kg} \mathrm{IBW}, p=0.049$, and $1.3 \pm 0.4 \mathrm{~g} /$ $\mathrm{kg}$ IBW vs $1.1 \pm 0.3 \mathrm{~g} / \mathrm{kg}$ IBW, $p=0.0019$, respectively]. Handgrip strength significantly increased after the program $(21.2 \pm 5.2 \mathrm{~kg}$ vs $20.0 \pm 5.3 \mathrm{~kg}, p=0.022)$. Likewise, gait speed and skeletal muscle mass index increased, although the differences did not reach statistical significance. Four patients became nonsarcopenic after the program. Postoperative complications were observed in
\end{abstract}

Kazuyoshi Yamamoto

kazuyoshi0208yamamoto@yahoo.co.jp

1 Department of Surgery, National Hospital Organization Osaka National Hospital, Osaka, Japan

2 Department of Surgery, NTT West Osaka Hospital, 2-6-40 Karasugatsuji, Tennoji-ku, Osaka 543-8922, Japan

3 Department of Food and Nutrition, National Hospital Organization Osaka National Hospital, Osaka, Japan

4 Department of Gastroenterological Surgery, Graduate School of Medicine, Osaka University, Osaka, Japan

5 Department of Surgery, Osaka General Medical Center, Osaka, Japan

6 Department of Surgery, Kaizuka City Hospital, Osaka, Japan three patients (13.6\%); however, none of these complications were severe (Clavien-Dindo grade III or lower). A preoperative exercise and nutritional support program has the potential to reduce sarcopenia and improve postoperative outcome in elderly sarcopenic patients with gastric cancer.

Keywords Stomach neoplasms $\cdot$ Sarcopenia $\cdot$ Preoperative care $\cdot$ Nutritional support $\cdot$ Exercise

\section{Introduction}

Sarcopenia was defined by Rosemberg [1] in 1989 as the attenuation of muscle mass and strength that is associated with aging. Sarcopenia was reported to be associated with postoperative complications and poor prognosis in various gastrointestinal cancer surgical procedures [2-5].

We previously reported [6] that sarcopenia was diagnosed in $21.2 \%$ of elderly gastric cancer patients undergoing gastrectomy according to the algorithm proposed by the European Working Group on Sarcopenia in Older People (EWGSOP) [7], and that preoperative calorie and protein intakes were significantly lower in sarcopenic patients than in nonsarcopenic patients. Furthermore, sarcopenia was identified as an independent risk factor for postoperative severe complications of Clavien-Dindo classification [8] grade III or higher.

To reduce the incidence of severe postoperative complications, in February 2014 we established a preoperative exercise and nutritional support program for elderly gastric cancer patients with sarcopenia undergoing gastrectomy. The aim of this study was to evaluate the efficacy of this program. 


\section{Methods}

\section{Patients}

Among 90 elderly gastric cancer patients aged 65 years or older who were scheduled to undergo gastrectomy from February 2014 to January 2016, sarcopenia was diagnosed in $22(24.4 \%)$ of them on the basis of the EWGSOP algorithm [7] at each patient's first visit to the outpatient clinic of the Department of Surgery, Osaka National Hospital (ONH). Specifically, patients underwent 4-m gait speed and handgrip strength testing, and we calculated the skeletal muscle mass index (SMI) by dividing skeletal muscle mass (determined by bioimpedance analysis) by height $\left(\mathrm{m}^{2}\right)$. The 22 sarcopenic patients were enrolled in the preoperative exercise and nutritional support program and were reevaluated after the program on the date of admission for surgery (Fig. 1).

\section{Preoperative exercise and nutritional support program}

This pilot study was approved by the Institutional Review Board of $\mathrm{ONH}$ and informed consent was

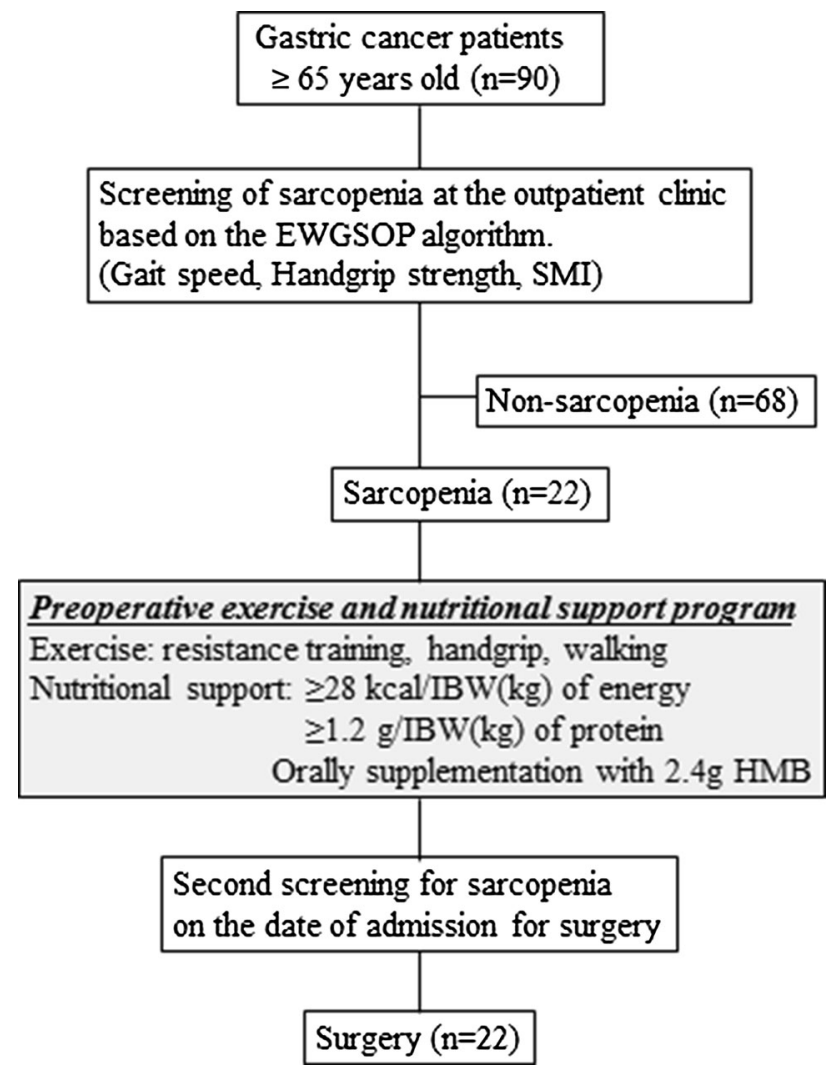

Fig. 1 Study schema. EWGSOP European Working Group on Sarcopenia in Older People, $H M B \quad \beta$-hydroxy- $\beta$-methylbutyrate, $I B W$ ideal body weight, $S M I$ skeletal muscle mass index obtained from all patients. The target duration of the preoperative program was 3 weeks, but the actual duration differed depending on the date of surgery. Patient self-report sheets were used to monitor adherence to the preoperative program and to provide motivation for participation.

\section{Exercise}

The preoperative exercise program consisted of handgrip training, walking, and resistance training. Handgrip training (10 kg) was done 20 times daily using each hand. Walking more than 7500 steps per day (about $1 \mathrm{~h}$ of walking) was recommended. Patients were required to perform three sets of ten repetitions of resistance training at $40-60 \%$ of one repetition maximum, in accordance with a previous report [9]. Resistance training comprised two patterns. Pattern A consisted of ten repetitions of push-ups, squats, and twisting sits-up. Pattern $\mathrm{B}$ involved ten repetitions of standing on tiptoes, straight sits-ups, and one arm and the opposite side leg up in all four positions. Patients alternated performing three repetitions of pattern A or pattern B at home every day until admission for surgery.

\section{Nutritional support}

All 22 enrolled patients were advised to aim for a total daily calorie intake of more than $28 \mathrm{kcal} / \mathrm{kg}$ ideal body weight (IBW), and a daily intake of protein of $1.2 \mathrm{~g} / \mathrm{kg}$ IBW, as well as $2.4 \mathrm{~g}$ daily oral supplementation with leucine metabolite $\beta$-hydroxy- $\beta$-methylbutyrate (HMB) in nutritional counseling. The recommended calorie and protein values to avoid sarcopenia were obtained from receiver operating characteristic analysis in our previous study [6]. Preoperative nutritional intake was calculated with use of a food frequency questionnaire by ONH registered dietitians at each patient's first visit to the outpatient clinic and at admission for surgery.

\section{Statistical analysis}

Continuous variables were expressed as the mean \pm standard deviation, except for the duration of the preoperative program, where the median and range were used. Fisher's exact test was used to compare categorical variables, and the paired $t$ test or Student's $t$ test was used to compare continuous variables. A $p$ value less than 0.05 was considered statistically significant. Statistical analysis was conducted with $\mathrm{JMP}^{\circledR}$ (SAS Institute, Cary, NC, USA). 


\section{Results}

\section{Patient characteristics}

The mean age of the 22 enrolled patients was 75 years and there were 10 men and 12 women. Fourteen patients underwent open gastrectomy and eight underwent laparoscopic gastrectomy. The numbers of patients with pathological stage I, II, III, and IV cancer according to the Japanese classification of gastric carcinoma (third English edition) [10] were $11,3,6$, and 2 respectively.

\section{Duration of and adherence to the preoperative exercise and nutritional support program}

The median duration of program participation was 16 days (range 7-26 days). No adverse events were observed during the program. The completion rates for resistance training and HMB supplementation were $50.0 \%$ and $54.5 \%$ respectively.

\section{Preprogram and postprogram nutritional intake, body composition, and sarcopenia parameters}

The total amounts of calories and protein were significantly increased after the program compared with those before $[29.4 \pm 6.9 \mathrm{kcal} / \mathrm{kg}$ IBW vs $27.3 \pm 5.6 \mathrm{kcal} / \mathrm{kg}$ IBW, $p=0.049, \Delta=(9.7 \pm 23.2) \%$, and $1.3 \pm 0.4 \mathrm{~g} / \mathrm{kg} \mathrm{IBW}$ vs $1.1 \pm 0.3 \mathrm{~g} / \mathrm{kg} \mathrm{IBW}, p=0.0019, \Delta=(25.4 \pm 39.3) \%$, respectively]. In terms of sarcopenia parameters, handgrip strength was significantly increased after the program $[21.2 \pm 5.2 \mathrm{~kg} \quad$ vs $\quad 20.0 \pm 5.3 \mathrm{~kg}, \quad p=0.022 ; \quad \Delta$ $=(8.1 \pm 18.6) \%]$. Gait speed and SMI were also increased $[0.85 \pm 0.22 \mathrm{~m} / \mathrm{s} \quad$ vs $\quad 0.80 \pm 0.21 \mathrm{~m} / \mathrm{s}, \quad p=0.064$, $\Delta=(10.3 \pm 25.7 \%)$, and $6.22 \pm 0.70 \mathrm{~kg} / \mathrm{m}^{2}$ vs $6.12 \pm$ $0.69 \mathrm{~kg} / \mathrm{m}^{2}, p=0.060, \Delta=(1.7 \pm 4.8) \%$, respectively]; however, these differences were not statistically significant. Of the 22 enrolled patients, four patients (18.2\%) were determined to be nonsarcopenic at the second screening (Table 1), and these four patients were all women.

\section{Duration of preoperative program and change in sarcopenia parameters and body composition}

We compared patients $(n=8)$ who participated in the preoperative program for 3 weeks or more with patients $(n=14)$ who participated for less than 3 weeks with regard to changes in sarcopenia parameters and body composition (Table 2). Increases in gait speed and handgrip strength were observed to some extent even in patients with the shorter duration of participation. Patients who participated for 3 weeks or more showed significant increase in lean body mass and an increase, though not significant, in SMI, compared with those who participated for less than 3 weeks.

\section{Postoperative clinical course}

Postoperative complications were observed in three patients (13.6\%). Grade II superficial surgical site infection was observed in two patients and grade II pneumonia was observed in one patient. No patients developed severe postoperative complications of Clavien-Dindo grade III or higher. In comparison, in nonsarcopenic elderly patients ( $n=68$, Fig. 1), the incidences of overall and severe postoperative complications were $13.2 \%$ and $2.9 \%$ respectively. For postoperative morbidity, comparable results were found between the two groups, even though the enrolled patients were older and tended to be more advanced complications than nonsarcopenic elderly patients (Table 3).

\section{Discussion}

Adequate calorie and protein intake and resistance training are key components in the management of sarcopenia $[11,12]$. In terms of the target duration of preoperative program, we hypothesized that 3 weeks of participation might be necessary to obtain meaningful changes in functional parameters and muscle mass when we established this program. One reason we considered a short-duration program is that the amount of time before surgery would be limited in this population for oncologic reasons. The other was that intensive nutritional support in addition to exercise might enhance recovery from sarcopenia. New metabolic agents such as vitamin D, leucine, and HMB have been reported to have an impact on muscle-related outcomes [13]. We adopted HMB supplementation in our program because randomized placebo-controlled trials demonstrated that HMB supplementation during resistance training effectively increased lean body mass and decreased muscle damage [14, 15].

Examination of the relationship between the duration of program participation and sarcopenic parameters revealed that gait speed and handgrip strength increased even after brief participation, whereas an increase in SMI was observed with participation duration longer than 3 weeks. These findings indicate that the rapid initial strengthening before the significant hypertrophy occurred in response to resistance training, which is consistent with a previous report [16].

To the best of our knowledge, this is the first report to evaluate the effectiveness of preoperative intervention for elderly sarcopenic patients with gastric cancer. We adopted 
Table 1 Preprogram and postprogram comparison of nutritional intake, body composition, and sarcopenia data $(n=22)$
Table 2 Changes in gait speed, handgrip strength, skeletal muscle mass index (SMI), and body composition according to the duration of the preoperative program

\begin{tabular}{llll}
\hline & Before program & After program & $p^{\mathrm{a}}$ \\
\hline Nutritional intake & & & \\
Amount of total calories (kcal/kg IBW) & $27.3 \pm 5.6$ & $29.4 \pm 6.9$ & 0.049 \\
Amount of protein (g/kg IBW) & $1.1 \pm 0.3$ & $1.3 \pm 0.4$ & 0.0019 \\
Body composition parameters & & & \\
Body mass (kg) & $54.7 \pm 8.7$ & $54.7 \pm 8.5$ & 0.98 \\
Fat mass (kg) & $16.2 \pm 6.3$ & $16.0 \pm 5.8$ & 0.75 \\
Lean body mass (kg) & $38.5 \pm 5.4$ & $38.7 \pm 5.4$ & 0.30 \\
Sarcopenia parameters & & & \\
Gait speed (m/s) & $0.80 \pm 0.21$ & $0.85 \pm 0.22$ & 0.064 \\
Handgrip strength $(\mathrm{kg})$ & $20.0 \pm 5.3$ & $21.2 \pm 5.2$ & 0.022 \\
SMI $\left(\mathrm{kg} / \mathrm{m}^{2}\right.$ ) & $6.12 \pm 0.69$ & $6.22 \pm 0.70$ & 0.060 \\
Sarcopenia patients $(n)$ & 22 & 18 & \\
\hline
\end{tabular}

Body weight body composition parameters were measured by bioimpedance analysis.

$I B W$ ideal body weight, $S M I$ skeletal muscle mass index

${ }^{\text {a }}$ Paired $t$ test

\begin{tabular}{|c|c|c|c|c|c|}
\hline & \multicolumn{2}{|c|}{$\geq 3$ weeks $(n=8)$} & \multicolumn{2}{|c|}{$<3$ weeks $(n=14)$} & \multirow[t]{2}{*}{$p$ value $^{\mathrm{a}}$} \\
\hline & Before & After & Before & After & \\
\hline \multirow[t]{2}{*}{ Gait speed $(\mathrm{m} / \mathrm{s})$} & $0.83 \pm 0.22$ & $0.94 \pm 0.21$ & $0.78 \pm 0.22$ & $0.81 \pm 0.23$ & \\
\hline & \multicolumn{2}{|c|}{$\Delta=(16.3 \pm 26.6) \%$} & \multicolumn{2}{|c|}{$\Delta=(6.9 \pm 25.5) \%$} & 0.43 \\
\hline \multirow[t]{2}{*}{ Handgrip strength $(\mathrm{kg})$} & $19.9 \pm 5.5$ & $21.4 \pm 6.4$ & $20.0 \pm 5.3$ & $21.1 \pm 4.7$ & \\
\hline & \multicolumn{2}{|c|}{$\Delta=(8.2 \pm 14.3) \%$} & \multicolumn{2}{|c|}{$\Delta=(8.0 \pm 21.1) \%$} & 0.98 \\
\hline \multirow[t]{2}{*}{ SMI $\left(\mathrm{kg} / \mathrm{m}^{2}\right)$} & $5.9 \pm 0.9$ & $6.2 \pm 0.9$ & $6.2 \pm 0.5$ & $6.3 \pm 0.6$ & \\
\hline & \multicolumn{2}{|c|}{$\Delta=(4.2 \pm 5.1) \%$} & \multicolumn{2}{|c|}{$\Delta=(0.4 \pm 4.2) \%$} & 0.10 \\
\hline \multirow[t]{2}{*}{ Fat mass $(\mathrm{kg})$} & $13.8 \pm 4.3$ & $13.6 \pm 3.7$ & $17.6 \pm 6.9$ & $17.4 \pm 6.4$ & \\
\hline & \multicolumn{2}{|c|}{$\Delta=(1.4 \pm 16.4) \%$} & \multicolumn{2}{|c|}{$\Delta=(-0.1 \pm 4.3) \%$} & 0.82 \\
\hline \multirow[t]{2}{*}{ Lean body mass $(\mathrm{kg})$} & $37.5 \pm 7.2$ & $38.4 \pm 7.3$ & $39.1 \pm 4.3$ & $38.8 \pm 4.2$ & \\
\hline & \multicolumn{2}{|c|}{$\Delta=(2.5 \pm 2.5) \%$} & \multicolumn{2}{|c|}{$\Delta=(-0.6 \pm 3.7) \%$} & 0.029 \\
\hline
\end{tabular}

a Student's $t$ test simple resistance training methods available to patients at home. For this reason, our preoperative program is readily applicable to any hospital and any type of cancer. The possible disadvantage of exercise at home is worsening of the preoperative condition by injury, but no adverse events occurred during program participation in 22 enrolled patients. In terms of the eligibility criteria, attenuation of muscle mass, strength, and physical performance associated with aging is a common problem for older people, and the definition of sarcopenia is matter of the cutoff value. We expect that our program may benefit all elderly patients undergoing surgery.

The current study has several limitations. This study was a pilot study and the sample size was small. The duration of the program was different on a case by case basis according to the date of surgery mainly because of the schedule of the operating theater. Additionally, the completion rate for resistance training of $50.0 \%$ was not extremely low but was unsatisfactory. Our preoperative exercise program might be tough for frailer patients, and exercising at home might be not always be done reliably, and then modification of the exercise program appropriate to each individual's muscle strength or exercise capacity will be needed to increase adherence to the program. To establish more conclusive evidence, a well-designed randomized controlled trial is necessary in which suitable end points would be decreases in the incidences of postoperative complications, functional recovery, length of hospital stay, quality of life, and improvement of prognosis, as well as reduction of sarcopenia. 
Table 3 Patient's backgrounds and incidence of postoperative complications among enrolled patients and nonsarcopenic patients

\begin{tabular}{|c|c|c|c|}
\hline & $\begin{array}{l}\text { Enrolled } \\
\text { patients } \\
(n=22)\end{array}$ & $\begin{array}{l}\text { Nonsarcopenic } \\
\text { patients } \\
(n=68)\end{array}$ & $p$ \\
\hline Age (years) & $75 \pm 5$ & $72 \pm 4$ & 0.041 \\
\hline Sex & & & 0.18 \\
\hline Male & 10 & 42 & \\
\hline Female & 12 & 25 & \\
\hline Surgical procedures & & & 0.15 \\
\hline OTG & 6 & 12 & \\
\hline ODG & 8 & 26 & \\
\hline OPG & 0 & 1 & \\
\hline LTG & 0 & 8 & \\
\hline LDG & 6 & 20 & \\
\hline LPG & 2 & 1 & \\
\hline Lymph node dissection & & & 0.61 \\
\hline $\mathrm{D} 1+$ & 10 & 37 & \\
\hline D2 & 11 & 30 & \\
\hline D3 & 1 & 1 & \\
\hline Operating time (min) & $265 \pm 70$ & $264 \pm 64$ & 0.97 \\
\hline Estimated blood loss (ml) & $170 \pm 192$ & $129 \pm 119$ & 0.37 \\
\hline Gastric cancer staging & & & 0.053 \\
\hline I-II & 14 & 57 & \\
\hline III-IV & 8 & 11 & \\
\hline All complications & $3(13.6 \%)$ & $9(13.2 \%)$ & 0.96 \\
\hline Superficial SSI & $2(9.1 \%)$ & $2(2.9 \%)$ & \\
\hline Pneumonia & $1(4.5 \%)$ & 0 & \\
\hline Leakage & 0 & $2(2.9 \%)$ & \\
\hline Pancreatic fistula & 0 & $2(2.9 \%)$ & \\
\hline Abdominal abscess & 0 & $1(1.5 \%)$ & \\
\hline Bleeding & 0 & $1(1.5 \%)$ & \\
\hline Heart failure & 0 & $1(1.5 \%)$ & \\
\hline $\begin{array}{l}\text { Severe complications ( } \geq \\
\text { grade III) }\end{array}$ & 0 & $2(2.9 \%)^{\mathrm{a}}$ & 0.29 \\
\hline
\end{tabular}

Severe complications are those of grade III or higher according to the Clavien-Dindo classification.

$L D G$ laparoscopic distal gastrectomy, $L P G$ laparoscopic proximal gastrectomy, $L T G$ laparoscopic total gastrectomy, $O D G$ open distal gastrectomy, $O P G$ open proximal gastrectomy, $O T G$ open total gastrectomy, SSI surgical site Infection

${ }^{a}$ In the nonsarcopenic group, the severe postoperative complications were abdominal abscess in one patient and heart failure in one patient.

In conclusion, our study suggests that a preoperative exercise and nutritional support program has the potential to reduce sarcopenia and improve postoperative clinical course in elderly sarcopenic patients with gastric cancer.

Acknowledgements The authors thank Akiko Toriyama, Chihiro Nakahara, and Tamaki Nakayama, national registered dietitians at Osaka National Hospital, for providing nutritional counseling and calculating calorie and protein intake. The authors also thank Takashi
Kitada, a physical therapist at Osaka National Hospital, for his support in designing the exercise program in this study.

\section{Compliance with the ethical standards}

Conflict of interest The authors declare that they have no conflict of interest.

Ethical standards All procedures followed were in accordance with the ethical standards of the responsible committee on human experimentation (institutional and national) and with the Helsinki Declaration of 1964 and later versions. Informed consent ore substitute for it was obtained from all patients for their being included in the study.

\section{References}

1. Rosenberg IH. Sarcopenia: origins and clinical relevance. J Nutr. 1997;127:990S-1S.

2. Ida S, Watanabe M, Yoshida N, Baba Y, Umezaki N, Harada K, et al. Sarcopenia is a predictor of postoperative respiratory complications in patients with esophageal cancer. Ann Surg Oncol. 2015;22:4432-7.

3. Reisinger KW, van Vugt JL, Tegels JJ, Snijders C, Hulsewé KW, Hoofwijk AG, et al. Functional compromise reflected by sarcopenia, frailty, and nutritional depletion predicts adverse postoperative outcome after colorectal cancer surgery. Ann Surg. 2015;261:345-52.

4. Joglekar S, Asghar A, Mott SL, Johnson BE, Button AM, Clark $\mathrm{E}$, et al. Sarcopenia is an independent predictor of complications following pancreatectomy for adenocarcinoma. J Surg Oncol. 2015;111:771-5.

5. Voron T, Tselikas L, Pietrasz D, Pigneur F, Laurent A, Compagnon $\mathrm{P}$, et al. sarcopenia impacts on short- and long-term results of hepatectomy for hepatocellular carcinoma. Ann Surg. 2015;261:1173-83.

6. Fukuda Y, Yamamoto K, Hirao M, Nishikawa K, Nagatsuma Y, Nakayama T, et al. Sarcopenia is associated with severe postoperative complications in elderly gastric cancer patients undergoing gastrectomy. Gastric Cancer. 2016;19:986-93.

7. Cruz-Jentoft AJ, Baeyens JP, Bauer JM, Boirie Y, Cederholm T, Landi F, et al. Sarcopenia: European consensus on definition and diagnosis: report of the European working group on sarcopenia in older people. Age Ageing. 2010;39:412-23.

8. Clavien PA, Barkun J, de Oliveira ML, Vauthey JN, Dindo D, Schulick RD, et al. The Clavien-Dindo classification of surgical complications: five-year experience. Ann Surg. 2009;250: 187-96.

9. Mattar MA, Gualano B, Perandini LA, Shinjo SK, Lima FR, SáPinto AL, et al. Safety and possible effects of low-intensity resistance training associated with partial blood flow restriction in polymyositis and dermatomyositis. Arthritis Res Ther. 2014;16:473.

10. Japanese Gastric Cancer Association. Japanese classification of gastric carcinoma: 3rd English edition. Gastric Cancer. 2011;14: $101-12$.

11. Morley JE, Argiles JM, Evans WJ, Bhasin S, Cella D, Deutz NE, et al. Nutritional recommendations for the management of sarcopenia. J Am Med Dir Assoc. 2010;11:391-6.

12. Peterson MD, Sen A, Gordon PM. Influence of resistance exercise on lean body mass in aging adults: a meta-analysis. Med Sci Sports Exerc. 2011;43:249-58.

13. Barillaro C, Liperoti R, Martone AM, Onder G, Landi F. The new metabolic treatments for sarcopenia. Aging Clin Exp Res. 2013;25:119-27. 
14. Panton LB, Rathmacher JA, Baier S, Nissen S. Nutritional supplementation of the leucine metabolite $\beta$-hydroxy- $\beta$-methylbutyrate $(\mathrm{hmb})$ during resistance training. Nutrition. 2000;16:734-9.

15. Nissen S, Sharp R, Ray M, Rathmacher JA, Rice D, Fuller JC Jr, et al. Effect of leucine metabolite $\beta$-hydroxy- $\beta$-methylbutyrate on muscle metabolism during resistance-exercise training. J Appl Physiol. 1996;81:2095-104.

16. Moritani T, deVries HA. Potential for gross muscle hypertrophy in older men. J Gerontol. 1980;35:672-82. 